\title{
Testagem anti-HIV: indagações sobre a expansão da oferta sob a perspectiva do acesso e da construção da demanda
}

The HIV diagnosis: questions about the supply broadening from the perspectives of access and demand construction

Neide Emy Kurokawa e Silva' , Luzia Aparecida Oliveira², Leyla Gomes Sancho³

1 Doutora em Ciências da Saúde pela Faculdade de Medicina da Universidade de São Paulo (USP) - São Paulo (SP), Brasil. Professora Adjunta do Instituto de Estudos em Saúde Coletiva, da Universidade Federal do Rio de Janeiro (UFRJ) - Rio de Janeiro (RJ), Brasil. neks@uol.com.br

2 Doutora em Ciências da Saúde pela Faculdade de Medicina da Universidade de São Paulo (USP) - São Paulo (SP), Brasil. Assistente Social do Serviço Ambulatorial Especializado em DST/AIDS Marcos Lottemberg, Prefeitura do Município de São Paulo - São Paulo (SP), Brasil. luzia.ao@uol.com.br

${ }^{3}$ Doutora em Saúde Coletiva pelo Instituto de Medicina Social da Universidade Estadual do Rio de Janeiro (UERJ) - Rio de Janeiro (RJ), Brasil. Professora Adjunta do Instituto de Estudos em Saúde Coletiva, da Universidade Federal do Rio de Janeiro (UFRJ) - Rio de Janeiro (RJ), Brasil. Isancho@uol.com.br
RESUMO: Reconhecendo a relevância do incremento na oferta de testagem anti-HIV, problematiza-se a concepção de oferta à luz dos conceitos de acesso e demanda. O mero foco na oferta da testagem mostra-se insuficiente frente à complexidade e dinamicidade do processo, desde a identificação da sua necessidade até a realização do teste e seus possíveis desdobramentos, incluindo o tratamento. Ademais, o modelo de campanha para incentivo à testagem não se coaduna com proposições que valorizem o protagonismo social dos cidadãos nem atendam às especificidades da disseminação da epidemia de AIDS. Postula-se a importância de desenvolver estudos que focalizem mais detidamente a demanda por testagem anti-HIV.

PALAVRAS-CHAVE: Síndrome da imunodeficiência adquirida; Diagnóstico HIV; Acesso; Necessidades e demandas de serviços de saúde.

ABSTRACT: Despite the relevance of the supply increase in anti-HIV testing, this article raises the problem behind the conception of supply in light of the concepts of access and demand. The supply of tests is in itself insufficient to handle the complexity and the dynamicity of a process that involves since the identification of its needs till the carrying out of the tests and their possible results, including the treatment. Furthermore, the campaign model designed to publicize testing is not consistent with the propositions that take into account the social role of individuals. This article brings to attention the importance of developing studies that focus on the demand for anti-HIV testing.

KEYWORDS: Acquired immunodeficiency syndrome; HIV diagnose; Access; Health services needs and demand. 


\section{Introdução}

\section{DO DIAGNÓSTICO TARDIO AO INÍCIO TARDIO DO TRATAMENTO DE AIDS: CONSIDERAÇÕES SOBRE O FOCO NA OFERTA DE TESTAGEM ANTI-HIV}

Dentre os componentes que caracterizam a chamada 'resposta brasileira à epidemia de AIDS', destaca-se o investimento programático, tanto nas açóes de prevenção ao HIV - como a distribuição de insumos de prevenção - como no tratamento de AIDS - especialmente por meio da política relativa à distribuição gratuita dos medicamentos antirretrovirais.

Um dos desafios a essa resposta, a despeito da disponibilização universal de serviços e dos medicamentos no Brasil, tem sido o início tardio do tratamento, ou seja, um número expressivo de pacientes apresenta contagem de células CD4 < 200 ou manifestações clínicas sugestivas de AIDS antes de seis meses da primeira consulta, o que pode comprometer sobremaneira o prognóstico e qualidade de vida dessas pessoas (SOUZA JUNIOR et al., 2006; FERNANDES et al., 2009).

O Relatório da UNGASS 2005-2007 (Sessão Especial da Assembleia-Geral das Naçóes Unidas) acerca da resposta brasileira ao HIV/AIDS indicava que, entre 2003 e 2006, 43,7\% das pessoas chegavam aos serviços de saúde já apresentando deficiência imunológica grave ou quadro clínico associado à AIDS (BRASIL, 2008b).

O diagnóstico tardio tem sido apontado como um dos principais fatores de risco para o início tardio de tratamento, cujos corolários são o desconhecimento do paciente quanto ao risco de infecção pelo HIV e a dificuldade de acesso à realização da testagem, conforme descrito por FERNANDES et al. (2009) a partir de estudos internacionais.

Além da preocupação com o adoecimento e risco de morte da pessoa infectada pelo HIV, postula-se que uma maior cobertura da testagem possibilitaria a interrupção da cadeia de transmissão do vírus a partir do pressuposto que a ciência do resultado reagente implicaria açóes preventivas (GAY; NAPRAVNIK; ERON JUNIOR, 2006).

Os primeiros testes anti-HIV no Brasil eram disponibilizados nos serviços de saúde voltados ao tratamento de pessoas vivendo com HIV por meio de serviços particulares. Motivados pelo aumento do número de casos e pelo forte preconceito associado à doença, os municípios de Porto Alegre e São Paulo foram pioneiros na criação dos então chamados Centros de Orientação e Apoio Sorológico (COAS), em 1989, expandindo-se posteriormente para o resto do País.

Em 1993, o Ministério da Saúde publicou documento intitulado "Normas de Organização e Funcionamento dos Centros de Orientação e Apoio Sorológico (COAS)", preconizando, dentre outras recomendaçóes, a realização do diagnóstico precoce de pessoas infectadas e de seus parceiros, a absorção de pessoas que procuravam os bancos de sangue para a realização do diagnóstico, além da oferta do aconselhamento, do preservativo e de informaçóes apropriadas e cientificamente embasadas.

Visando à garantia de maior aporte emocional aos indivíduos que procuram os COAS, estes passaram a incorporar o trabalho de aconselhamento, justificando a mudança da denominação, em 1997, para Centro de Testagem e Aconselhamento (CTA).

Nesse período, as mudanças na dinâmica da epidemia, tais como o crescimento do número de casos entre heterossexuais, mulheres e população de baixa renda; a descoberta de novos medicamentos para o tratamento da AIDS e a possibilidade de prevenir a transmissão vertical do HIV; e a consolidação do Sistema Único de Saúde, fundamentado na atenção primária e na descentralização da política de saúde, impuseram novas modificaçôes na oferta do teste anti-HIV. A possibilidade de prevenir a transmissão vertical do HIV, por exemplo, ampliou a oferta da testagem para os serviços de atenção à saúde pré-natal.

Ao lado do novo cenário, convivia-se com a manutenção das altas taxas de prevalência da doença entre os grupos sociais historicamente afetados pela epidemia, como homens que fazem sexo com homens, usuários de drogas e profissionais do sexo, cabendo aos CTAs incorporar tanto a universalização do diagnóstico do HIV quanto garantir a equidade no acesso aos serviços das populaçóes mais vulneráveis.

Diante desse quadro, no Brasil, têm-se privilegiado estratégias visando a ampliar a cobertura da 
testagem anti-HIV por meio do investimento na capacidade dos CTAs, do recurso ao teste rápido para detecção da infecção pelo HIV e do investimento em campanhas dirigidas à população em geral, como o 'Fique Sabendo' ou o 'Quero Fazer'. Especialmente em relação aos CTAs, o Brasil, segundo estudo publicado em 2008, conta com 383 unidades cujas implantaçóes nas diferentes regiōes do país seguiram a evolução da epidemia (BRASIL, 2008a).

Não obstante a relevância dessas iniciativas, indaga-se a ênfase em estratégias ancoradas, sobretudo, na preocupação com a oferta de recursos e serviços, a partir do pressuposto que a população não estaria sendo testada por desconhecer ou não contar com esses serviços.

Até o momento, não se dispóe de estudos que examinem mais detida e especificamente as iniciativas de expansão da testagem, mas investigação conduzida pelo Ministério da Saúde-CEBRAP mostra que cerca de $30 \%$ da população brasileira sexualmente ativa havia realizado ao menos um anti-HIV na vida. Mesmo considerando que a cobertura desejável seja próxima de $100 \%$, estes autores julgam relativamente elevada a proporçáo de brasileiros que relatou ter sido testada (FRANÇA JUNIOR; CALAZANS; ZUCCHI, 2008).

Em relação a grupos específicos, a proporção de homens que fazem sexo com homens, por exemplo, é maior do que a de homens que fazem sexo exclusivamente com mulheres (33\% e 21\%, respectivamente). O Plano Nacional de Enfrentamento da Epidemia de AIDS e das DST entre Gays, Homens que fazem Sexo com Homens (HSH) e Travestis registra que esses índices, entretanto, são inferiores a outros países que promoveram estratégias de acesso universal ao diagnóstico e ao tratamento (BRASIL, 2007), reportando o problema na cobertura da testagem como sendo consequência da dificuldade no acesso a serviços de saúde.

Essa é uma noção que tem permeado os discursos sobre início tardio do tratamento, justificando-o como consequência de diagnóstico tardio motivado por problemas no acesso; este, por sua vez, traduzido exclusiva ou privilegiadamente como problemas na oferta de serviços e na disponibilização do teste anti-HIV.

Compartilha-se a importância de mitigar os impactos adversos decorrentes do início tardio do tratamento; no entanto, destaca-se a necessidade de ampliar o escopo das análises e intervençôes calcadas exclusivamente na lógica de que os indivíduos querem ser testados, bastando disponibilizar serviços que atendam a essa demanda.

Muitos contextos concorrem para que as pessoas tomem a iniciativa de ser testadas, iniciem e deem continuidade ao tratamento: entre a percepção da necessidade, a motivação para realizar o teste de HIV e a busca por tratamento transita uma rede de valores, temores, atores que pode comprometer os 'desfechos' esperados frente a resultados reagentes.

Nesse sentido, parece oportuno problematizar a centralidade da ampliação ou expansão da oferta, resgatando e cotejando-a com referências conceituais sobre 'acesso', posto que, embora a oferta possa fazer parte do entendimento sobre acesso, os termos não se equivalem.

O conceito de acesso permite um exame mais abrangente, que extrapola o registro numérico de pessoas testadas em determinado espaço de tempo e geográfico, incluindo, dentre outros, a relação entre serviços de saúde e usuários e a própria construção da demanda, no caso, por testagem anti-HIV.

O objetivo do presente ensaio é discutir os distintos desdobramentos teóricos e práticos entre oferta de testagem e acesso à testagem anti-HIV, explorando a fecundidade conceitual deste último na compreensão do diagnóstico tardio de HIV.

\section{Metodologia}

Sem a pretensão de proceder a uma revisão dos trabalhos sobre o assunto, selecionaram-se publicaçóes que contribuíssem para a problematização da ideia de oferta subjacente às estratégias de expansão da testagem anti-HIV. Não havendo resultados para termos como 'expansão' ou 'oferta' do teste anti-HIV, a busca nas bases Medline, Lilacs e SciELO pautou-se nos termos: 'acesso', 'teste', 'testagem' e 'HIV'.

Os trabalhos nacionais versando sobre o tema concentram-se no âmbito dos CTAs ou da atenção primária e suas capacidades de produção; perfil dos usuários; ou cobertura desse procedimento junto às gestantes, além 
de consideraçóes sobre o teste rápido (GRANGEIRO et al., 2009a,b; SOUZA; CZERESNIA, 2010; VELOSO et al., 2008, 2010; CARDOSO et al., 2007; TELLES-DIAS et al., 2007).

Apenas um dos estudos aproximou-se especificamente da temática da expansão da testagem (FRANÇA JUNIOR; CALAZANS; ZUCCHI, 2008), fornecendo o substrato inicial de informaçóes a partir das quais se desenvolveu este artigo. O cotejamento com os outros estudos visou ressaltar elementos que propiciassem o exame das estratégias de ampliação da oferta de testagem à luz das perspectivas de acesso afinadas com a complexidade e multidimensionalidade do processo que envolve a identificação de necessidades, a construção da demanda e a oferta da atenção à saúde.

Nesse sentido, a análise do material baseou-se nos conceitos de acesso e de construção social da demanda com o objetivo de captar tal complexidade e multidimensionalidade a partir de tratamento interpretativo-compreensivo (MINAYO, 1993), tendo como eixo organizador o quadro conceitual da vulnerabilidade (AYRES et al, 1999, 2003, 2006).

\section{O conceito de acesso e suas múltiplas possibilidades de apreensão}

Se, por um lado, a abrangência do conceito de acesso pode ser tomada como um obstáculo pela imprecisão do termo (ADAY; ANDERSEN, 1974) e dificuldade de apreendê-lo objetivamente, por outro, pode expressar as potencialidades dessa abrangência, permitindo extrapolar a simples dimensão da oferta de serviços mesmo considerando que, a depender da abordagem, possa subsumir-se a este significado.

O recurso aos Descritores em Ciências da Saúde (DeCS) já enuncia a extensão do termo, que indica desde a acessibilidade aos serviços e cuidados de saúde e a qualidade e avaliação do processo que envolve o acesso até a equidade no acesso, visando a oferecer alternativas para os indivíduos que teriam maior dificuldade em adentrar e utilizar os serviços de saúde.

Dentre as possibilidades de apreensão, o conceito de acesso pode enfocar as características dos indivíduos, da oferta, de ambos ou a relação dos indivíduos com os serviços de saúde ou, ainda, tomado em sua dimensão de acessibilidade, evidenciar o ato de ingressar no sistema e o grau de facilidade com que as pessoas obtêm cuidados de saúde.

Vale ressaltar, entretanto, que a integração antevista para as diferentes dimensóes do acesso tende a ter caráter mecanicista, de ajustamento entre diferentes etapas do processo de utilização dos serviços (TRAVASSOS; MARTINS, 2004). Este, por sua vez, estaria calcado na oferta de serviços e na otimização dessa oferta sob as lógicas de custo-benefício ou de custo-efetividade, que, de certo modo, excluiriam do processo os destinatários das intervençóes, tratados como consumidores de serviço.

A ampliação da oferta de testagem tem sido desenvolvida por meio de campanhas que incentivam a sua realização, disponibilizando-a, inclusive, em locais com grande concentração de público, como ocorrido em 2012 no Rock in Rio, grande evento musical que reuniu milhares de pessoas, levando a indagar sobre a qualificação dessa oferta, ou seja, o quanto ela estaria sintonizada com as características da demanda por esse serviço ou mesmo com as reais chances de tratamento, tomando o acesso em sua perspectiva mais abrangente.

Registra-se que, de 1999 a 2005, a expansão da testagem atingiu principalmente pessoas com melhores condiçóes sociais e mulheres em idade reprodutiva, adultas, estas últimas, possivelmente, em função da obrigatoriedade da sua oferta durante o pré-natal desde 1996. Questiona-se o caráter voluntário da testagem nesse contexto, além da necessidade de expansão proporcional na provisão de aconselhamento (FRANÇA JUNIOR; CALAZANS; ZUCCHI, 2008).

$\mathrm{O}$ aconselhamento, a despeito de suas contradiçóes nas práticas de saúde envolvendo a realização da testagem (PUPO, 2007), grosso modo, é o espaço para que o indivíduo possa externar suas preocupações, obter informaçóes, ser orientado por um profissional de saúde na avaliação de possíveis situaçóes de vulnerabilidade à infecção pelo HIV para que tenha condiçóes de decidir autonomamente sobre seu desejo e pertinência de realizar a testagem. 
Ainda tomando o Rock in Rio como exemplo, quais as chances de realização de aconselhamento em condiçóes de privacidade em um espaço como esse? Quais as consequências do recebimento de um resultado reagente nesse contexto? Quais as possibilidades de fazer a vigilância para eventuais casos reagentes?

Com uma proposta mais abrangente de acesso, Andersen (1995) incorpora o que denomina etapa de utilização de serviços, que seria mediada por fatores predisponentes existentes existem antes do surgimento do problema de saúde e que afetam a predisposição das pessoas para usar o serviço de saúde; fatores capacitantes, que seriam os meios disponíveis para que as pessoas recebessem cuidados de saúde; necessidades de saúde, como as condiçôes de saúde percebidas pelas pessoas ou diagnosticadas por profissionais de saúde.

O autor postula dois elementos do acesso, que são o acesso potencial, que diz respeito a fatores individuais que limitam ou ampliam a capacidade de uso e o acesso realizado, que inclui os fatores predisponentes, as necessidades de saúde e o que chama de fatores contextuais, atinentes às políticas de saúde e à oferta de serviços.

Dessas proposiçóes terminológicas, depreende-se a preocupação de vislumbrar as diferentes dimensões capazes de afetar as diferentes etapas, desde a demanda por um serviço de saúde até a oferta propriamente dita.

Penchansky e Thomaz (1981) também ampliam o âmbito da oferta, identificando dimensóes que comporiam o conceito de acesso: disponibilidade, volume e tipo de serviços em relação às necessidades; acessibilidade, caracterizada pela adequação geográfica entre serviços de saúde e usuários; acolhimento, que focaliza o contato entre a organização dos serviços de saúde e a capacidade dos usuários de adaptar-se a essa organização; capacidade de compra, a respeito das formas de financiamento dos serviços e capacidade de as pessoas pagarem por esses serviços; aceitabilidade, que representa a atitude dos usuários e profissionais de saúde em relação às características e práticas de cada um.

Uma olhada inicial nas citadas abordagens sobre acesso permite problematizar o fato de que uma das açóes estratégicas privilegiadas para o controle da epidemia de AIDS tem sido a expansão da testagem anti-HIV, ancorada em estratégias de campanhas publicitárias.
Estudos brasileiros sobre o tema indicam que o percentual de pessoas testadas acompanha uma tendência internacional e que há distinçóes no padrão de testagem em diferentes estratos da população, ilustradas pelo fato de a cobertura de testagem ser menor dentre os menos escolarizados, moradores de regióes mais pobres, de regióes não metropolitanas e pessoas de classes sociais menos favorecidas (FRANÇA JUNIOR; CALAZANS; ZUCCHI, 2008; SCHNEIDER et al., 2008; BRASIL, 2008a).

As campanhas ou incentivo à realização da testagem anti-HIV nem sempre logram o sucesso esperado em alguns segmentos, mormente naqueles em que a incidência do HIV tem sido mais expressiva, a exemplo dos homens que fazem sexo com homens. Entretanto, não se pode afirmar que, por si só, o fato de estarem inseridos em certos grupos determinaria a busca ou recusa da testagem ou mesmo o início do tratamento, mas os contextos sociais e culturais que modelam as relaçóes, os valores, os afetos e mesmo a noção de direitos entre os indivíduos.

Não estando as pessoas alheias ao risco de infecção pelo HIV nem desconhecendo os meios para realizar a testagem, o problema náo pode ser tomado genericamente como uma questão de divulgação e de disponibilidade de insumos e serviços. $\mathrm{O}$ desafio parece residir na compreensão das necessidades desses grupos, conformadas como necessidades de saúde e, consequentemente, das possibilidades e limites para transformarem-se em demandas para os serviços de saúde.

\section{Demanda por testagem anti-HIV como construção social}

Inspirados na clássica conceituação de demanda como 'quantidade de bem ou serviço que as pessoas desejam consumir', os modelos explicativos sobre demandas no campo da saúde são comumente ancorados em referenciais da economia e do planejamento em saúde no campo da saúde. Nesse sentido, apoiam-se em uma estrutura de preferências ou de desejos de um consumidor (IUNES, 1995). 
A despeito da banalização da expressão construçáo social (CAMARGO JUNIOR, 2005), parece pertinente recorrer a ela, no sentido de possibilitar uma apreensão das demandas em saúde de forma contextualizada e historicamente situada.

Ao propor uma estratégia de expansão da oferta de teste, deduz-se a identificação de necessidade de saúde, expressa tanto pela demanda dos usuários - que avaliam ter se engajado em alguma situação de risco, por exemplo, como pelas fontes tradicionais que embasam as ações de planejamento em saúde. O raciocínio lógico levaria a crer em um processo linear que culminaria no diagnóstico e tratamento precoces, evitando o adoecimento e possibilitando maior sobrevida do indivíduo soropositivo para o HIV.

Tomar a demanda por testagem como uma construção social exige o cuidado em não torná-la mais um bordão genérico, esvaziado de referências conceituais (CAMARGO JUNIOR, 2005).

No tema em pauta, um primeiro aspecto decorrente dessa exigência diz respeito à compreensão das desigualdades no perfil da população que busca a testagem ou, sob outro prisma, daqueles que não conseguem acessá-la.

Num polo, privilegiam-se as características e capacidades individuais que subsumiriam a iniciativa de realizar a testagem a uma questão de 'opçáo' pessoal. Tal perspectiva costuma coadunar-se com a premissa que, de posse de informaçóes adequadas, os indivíduos seriam 'conscientizados' da importância de realizar a testagem. Sob a mesma lógica, as pessoas que, mesmo tendo justificativas para realizar a testagem não o fazem, seriam consideradas culpadas pelo fato de se exporem ao adoecimento e, consequentemente, exporem outros ao risco de infecção.

Os estudos e práticas no campo da AIDS têm protagonizado a crítica às abordagens que imputam a responsabilidade pela infecção ou transmissão do HIV aos indivíduos, e o conceito de vulnerabilidade vem justamente incorporar ativamente outras dimensóes da epidemia que concorrem para suscetibilizar ou proteger as pessoas e populaçóes contra o HIV (AYRES et al., 1999, 2003, 2006). Paralelamente à dimensão individu$a l$, que envolve as capacidades e fragilidades das pessoas nas possibilidades de prevenção, incluindo crenças, valores, interesses e redes e suportes sociais, destacam-se a dimensão programática - concernente às açóes institucionais para o controle da epidemia, compromissos políticos dos governos e definição de políticas específicas - e a dimensão social, na qual se ressaltam as normas sociais, relaçóes de gênero, raça/etnia, normas e crenças religiosas, dentre outras (AYRES et al., 2006).

Nesse sentido, a realização da testagem não estaria afeta à vontade dos indivíduos ou à sua disponibilização, mas envolveria uma miríade de situaçóes que culminariam nessa iniciativa, bem como nas etapas subsequentes de busca e adesão por tratamento.

No bojo da articulação entre as dimensões programática e social, outra abordagem possível para a desigualdade no acesso pode seguir o debate sobre equidade na atenção à saúde.

No cenário do Sistema Único de Saúde (SUS), um dos desafios à noção de acesso diz respeito ao seu berço, que é o planejamento em saúde, especialmente no tocante à lógica econômica, visando ao equilíbrio na relação entre demanda e oferta, ou seja, informada pela racionalidade mercadológica (JESUS; ASSIS, 2010).

Para fazerem face a esse paradoxo que envolve a equidade no acesso à saúde e a lógica economicista, têm sido propostos dispositivos tecnológicos capazes de superar as adversidades impostas pelo modelo neoliberal. Um deles seria a realização de inquéritos epidemiológicos em saúde, que permitiriam identificar, além da disponibilidade de serviços e recursos, a acessibilidade, a acomodação ou adequação funcional dos serviços, a capacidade financeira e a aceitabilidade dos usuários. Em outros termos, os inquéritos de saúde possuiriam uma 'ação demandante' sobre os serviços, captando necessidades não explicitadas pelos usuários, nomeadas como necessidades invisíveis (JESUS; ASSIS, 2010).

Não cabe, aqui, problematizar a ideia de necessidades invisíveis, que, de certo modo, choca com a dinamicidade que se pretende tomar por meio das noçóes de acesso e de construção social da demanda, já que elas não seriam preexistentes e já conformadas, à espera de uma intervenção, mas parece adequado incorporar a voz daqueles sujeitos que não acessaram a testagem, a 
partir das barreiras pessoais, programáticas e sociais que se interporiam à sua realização.

O segundo aspecto que a ideia de construção social da demanda apresenta diz respeito às finalidades da ênfase na ampliação da testagem que apostam nos procedimentos técnicos, visando tanto ao controle da cadeia de transmissão do HIV como ao tratamento precoce, a fim de evitar o adoecimento do indivíduo.

A ênfase nas finalidades técnicas é caudatária da sua identificação com a dimensão biomédica da atenção à saúde, fundada em critério normativo de êxito que visa ao controle de riscos, à correção de uma disfunção ou dismorfia ou ao restabelecimento de um bem-estar físico ou psíquico (AYRES, 2001). Nessa concepção, a antevisão do controle técnico sobrepóe-se às perspectivas e obstáculos práticos, concernentes "às aspirações cotidianas dos usuários dos serviços, relacionadas à saúde, mas também e especialmente à vida de forma mais ampla, ao bem viver de modo geral" (OLIVEIRA et al.; AYRES, 2005, p.691).

É quase 'automática' a associação entre oferta de testagem e utilização desse recurso pela população dados os possíveis benefícios que isso acarretaria sob os pontos de vista da prevenção e do tratamento, como discutido por Ayres:

Quando pensamos na assistência à saúde, vem-nos de imediato à mente a aplicaçấo de tecnologias para o bem estar físico e mental das pessoas. Em geral a formulação é simples: a ciência produz o conhecimento sobre as doenças, a tecnologia transforma esse conhecimento em saberes e instrumentos para a intervenção, os profissionais de saúde aplicam esses saberes e instrumentos e produz-se a saúde. Precisamos considerar que a direção inversa também é verdadeira: que o modo como aplicamos e construimos tecnologias e conhecimentos cientificos determina limites para o que podemos enxergar como necessidades de intervenção em saúde. (AYRES, 2004, p.84).

$\mathrm{O}$ autor prossegue, chamando a atenção para o fato que o modo como as tecnologias e conhecimentos científicos são construídos pode afetar nossa capacidade de compreensão das necessidades de saúde:

Precisamos ter claro também que nem tudo que é importante para o bem estar pode ser imediatamente traduzido e operado como conhecimento técnico. E por fim, mas fundamental, precisamos estar atentos para ofato de que nunca, quando assistimos à saúde de outras pessoas, mesmo estando na condição de profissionais, nossa presença na frente do outro se resume ao papel de simples aplicador de conhecimentos. Somos sempre alguém que, percebamos ou não, está respondendo a perguntas do tipo: "O que é bom pra mim?", "Como devo ser?", "Como pode ser a vida?” (AYRES, 2004, p.84).

Ainda que a aspiração ao êxito técnico seja pregnante nas práticas de saúde, levando, muitas vezes, à priorizaçáo inconteste do controle da doença, a despeito das aspiraçóes e dos horizontes e significados do bem viver das pessoas, parece apropriado, no caso da testagem anti-HIV, que esse procedimento seja tomado no contexto dessas aspiraçóes e horizontes.

A reflexão do autor pode referenciar o debate sobre o modo como a questáo da testagem anti-HIV vem sendo tratada: ainda que a ampla oferta possa ser interpretada como modo de disseminar esse serviço à população geral, subjaz no seu modus operandi a identificação com as campanhas, de ímpeto sanitarista, passando ao largo dos contextos particulares e das aspiraçóes cotidianas que propiciariam, ou não, a realização do diagnóstico sorológico.

Após situar-se a noção de ampliação do diagnóstico no escopo dos conceitos de acesso e de demanda como construção social, torna-se oportuno pontuar dois aspectos diretamente afetos ao objeto do estudo, relativos às relaçôes estabelecidas entre indivíduos e populaçôes e às ações de saúde, especialmente a oferta de testagem anti-HIV e o papel do Aconselhamento nessa oferta, considerando que, originalmente, essa atenção era inerente à testagem em si. 


\section{Das campanhas à problematização da testagem anti-HIV}

A despeito do pioneirismo das políticas voltadas para atenção à AIDS no País, que privilegiam o protagonismo e participação sociais em estreita consonância com os princípios do SUS, incluindo a integralidade da atenção, a proposta de expansão da oferta de testagem parece reproduzir os modelos das campanhas, inclusive voltando-se para a responsabilização do indivíduo na decisão de realizar o teste.

Ainda que o impacto da imagem do homem 'carregando um piano', que serviu de mote para uma das campanhas, possa ter feito com que as pessoas tenham se identificado com o 'peso' da incerteza de estar infectado pelo HIV, não se sabe o quanto esse peso propiciou a busca efetiva pela testagem.

A incitação à realização do teste, ainda que mais atenuada que os moldes compulsórios das históricas campanhas sanitárias, assemelha-se a elas, mas, desta feita, travestida como propaganda que não impóe, mas busca 'seduzir' o indivíduo para que procure um serviço de saúde, a fim de fazer o exame.

A distinção entre sujeitos-consumidores de serviços e sujeitos-cidadãos vem sendo apontada por Paiva (2002) tanto no trabalho de prevençáo como no cuidado à saúde no contexto do HIV. A autora alerta para a diferença entre oferecer um produto pronto para o consumo e as possibilidades de construção conjunta desse produto, a partir da interação entre os agentes - usuários e profissionais de saúde - e de tematizar e problematizar, no caso, a realização ou não da testagem.

Um dos espaços privilegiados para essa problematização tem sido o chamado aconselhamento sorológico, que, como já dito, é alvo de intensos debates tanto em relação à sua consistência conceitual como à qualidade de sua prática (PUPO, 2007; GRANGEIRO et al., 2009).

Em que pese esse debate, parece certo que espaços como o aconselhamento sorológico não só devem ser qualificados como, sobretudo, deve-se incentivar a criação de novas estratégias que possibilitem a reflexão e explicitação das possibilidades e limites à realização da testagem.

Sob as proposiçóes do referencial da vulnerabilidade, foi possível, de certo modo, rever os discursos e práticas em torno dos chamados grupos e comportamentos de risco, flagrantes equívocos que impregnaram o discurso epidemiológico com vieses morais. Sob uma perspectiva distinta e mais cuidadosa, apontam-se as 'epidemias concentradas', tais como as apresentadas no trabalho de Barbosa Junior et al. (2009). Assim, ainda que a epidemia esteja concentrada, por exemplo, em grupos de profissionais do sexo, as ações não precisariam ser exclusivamente focalizadas nessa população, recorrendo-se a outros elementos-chave, como os clientes desses profissionais do sexo.

Aliada à inovação de estratégias que possam ir ao encontro das necessidades da população, parece pertinente, diante da carência de estudos específicos sobre o assunto no Brasil, a condução de investigaçóes que propiciem o aprofundamento da compreensão da construção da demanda por testagem anti-HIV, numa perspectiva contextualizada social e culturalmente, aberta a alternativas teóricas e metodológicas. As possíveis barreiras que dificultam a realização da testagem anti-HIV, quem sabe, coincidem com aquelas que levariam as pessoas já diagnosticadas a buscarem tardiamente o tratamento.

\section{Referências}

ADAY, L.A.; ANDERSEN, R. A framework for the study of access to medical care. Health Services Research, v.9, n.3, p.208-220, 1974.
ANDERSEN, R.M. Revisiting the behavioral model and access to medical care: does it matter? Journal of Health and Social Behavior, v.36, p.1-10, 1995. 
AYRES, J.R.C.M. Cuidado e reconstrução das práticas de saúde. Interface: Comunicação, Saúde, Educação, v.8, n.14, p.73-91, 2004.

Sujeito, intersubjetividade e práticas de saúde. Ciência e Saúde Coletiva, v.6, n.1, p.63-72, 2001.

AYRES, J.R.C.M. et al. O conceito de vulnerabilidade e as praticas de saúde: novas perspectivas e desafios. In: CZERENIA, D.; FREITAS, C.M. (Org). Promoção da Saúde: conceitos e tendências. Rio de Janeiro: FIOCRUZ, 2003. p.117-139.

Risco, vulnerabilidade e práticas de prevenção e promoção da saúde. In: CAMPOS, G.W.S. et al. (Orgs.). Tratado de saúde coletiva. São Paulo: Hucitec; Rio de Janeiro: FIOCRUZ, 2006. p.375418.

Vulnerabilidade e prevenção em tempos de AIDS. In: PARKER, R.; BARBOSA, R.M. (Orgs). Sexualidades pelo avesso: direitos, identidades e poder. São Paulo: Editora 34, 1999. p.49-71.

BARBOSA JUNIOR, A. et al. Tendências da epidemia de AIDS entre subgrupos sob maior risco no Brasil, 1980-2004. Cadernos de Saúde Pública, v.24, n.4, p.727-737, 2009.

BRASIL. Ministério da Saúde. Secretaria de Vigilância em Saúde. Programa Nacional de DST e AIDS. Contribuição dos centros de testagem e aconselhamento para universalizar o diagnóstico e garantir a equidade no acesso aos serviços. Ministério da Saúde, Secretaria de Vigilância em Saúde, Programa Nacional de DST e AIDS. Brasília: Ministério da Saúde, 2008a.

UNGAS. Resposta Brasileira 2005-2007. Relatório de Progresso do Pais. Ministério da Saúde. Secretaria de Vigilância em Saúde. Programa Nacional de DST e AIDS. Brasília: Ministério da Saúde, $2008 b$.

Plano Nacional de Enfrentamento da epidemia de AIDS e das DST entre Gays, HSH e Travestis. Brasília, 2007.

CAMARGO JUNIOR, K.R. Das necessidades de saúde à demanda socialmente constituída. In: PINHEIRO, R.; MATTOS, R.A. (Org.). Construção Social da Demanda. Rio de Janeiro: IMS/UERJ/CEPESC/ ABRASCO, p.93-101, 2005.
CARDOSO, A.J. et al. Infecção pelo HIV entre gestantes atendidas nos centros de testagem e aconselhamento em AIDS. Revista de Saúde Pública, v. 41, Suplemento 2, p.101-108, 2007.

FERNANDES, J.R.M. et al. Início de terapia antirretroviral em estágio avançado de imunodeficiência entre indivíduos portadores de HIV/AIDS em Belo Horizonte, Minas Gerais, Brasil. Cadernos de Saúde Pública, v.25, n.6, p.1369-1380, 2009.

FRANÇA JUNIOR, I.; CALAZANS, G.; ZUCCHI, E.M. Grupo de Estudos em População, Sexualidade e AIDS. Mudanças no âmbito da testagem anti-HIV no Brasil entre 1998 e 2005. Revista de Saúde Pública, v.42, Suplemento 1, p.84-97, 2008.

GAY, C.L.; NAPRAVNIK, S.; ERON JUNIOR, J.J. Advanced immunosuppression at entry to HIV care in the south-eastern United States and associated risk factor. AIDS, v.20, n.5, p.775-778, 2006.

GRANGEIRO, A. et al. Voluntary counseling and testing (VCT) services and their contribution to access to HIV diagnosis in Brazil. Cadernos de Saúde Pública, v.25, n.9, p.2053-2063, 2009.

Avaliação do perfil tecnológico dos centros de testagem e aconselhamento para HIV no Brasil. Revista de Saúde Pública, v.43, n.3, p.427-436, 2009.

IUNES, R.E. Demanda e demanda em Saúde. In: PIOLA, S.E.; VIANA, S.M. (Orgs). Economia da Saúde: conceitos e contribuições para gestão da saúde. Brasil: IPEA, 1995.

JESUS, W.L.A.; ASSIS, M.M.A. Revisão sistemática sobre o conceito de acesso nos serviços de saúde: contribuição do planejamento. Ciência \& Saúde Coletiva, v.15, n.1, p.161-70, 2010.

MINAYO, M.C.S. O desafio do conhecimento: pesquisa qualitativa em saúde. São Paulo: Editora Hucitec; Rio de Janeiro: ABRASCO, 1993.

OLIVEIRA, L.A. et al. Humanização e cuidado: a experiência da equipe de um serviço de DST/AIDS no município de São Paulo. Ciência e Saúde Coletiva, v.10, n.3, p.689-698, 2005.

PAIVA, V. Sem mágicas soluções: a prevenção e o cuidado em HIV/ AIDS e o processo de emancipação psicossocial. Interface-Comunicação, Saúde, Educação, v.6, n.11, p.25-38, 2002. 
PENCHANSKY, D.B.A.; THOMAS, J.W. The concept of access - definition and relationship to consumer satisfaction. Medical Care, v.19, n.2, p.127-140, 1981.

PUPO, L.R. Aconselhamento em DST/AIDS: uma análise crítica de sua origem histórica e conceitual e de sua fundamentação teórica. Dissertação (Mestrado em Medicina Preventiva) - Universidade de São Paulo, São Paulo, 2007.

SCHNEIDER, I.J. et al. Perfil epidemiológico dos usuários dos Centros de Testagem e Aconselhamento do Estado de Santa Catarina, Brasil, no ano de 2005. Cadernos de Saúde Pública, v.24, n.7, p.16751688, 2008.

SOUZA, V.S.; CZERESNIA, D. Demandas e expectativas de usuários de centro de testagem e aconselhamento anti-HIV. Revista de Saúde Pública, v.44, n.3, p.441-447, 2010.

SOUZA-JR, P.R.B.; SZWARCWALD, C.L.; CASTILHO, E.A. Delay in introducing antiretroviral therapy in patients infected by HIV in Brazil, 2003-2006. Clinics, v.62, n.5, p.579-84, 2007.

TELLES-DIAS, P.R. et al. Impressões sobre o teste rápido para o HIV entre usuários de drogas injetáveis no Brasil. Revista de Saúde Pública, v.41, suppl. 2, p.94-100, 2007.
TRAVASSOS, C.; MARTINS, M. Uma revisão sobre os conceitos de acesso e utilização de serviços de saúde. Cadernos de Saúde Pública, v.20, suppl. 2, p.190-198, 2004.

VELOSO, V.G. et al. HIV rapid testing as a key strategy for prevention of mother-to-child transmission in Brazil. Revista de Saúde Pública, v.44, n.5, p.803-811, 2010.

VELOSO, V.G. et al. HIV testing among pregnant women in Brazil: rates and predictors. Revista de Saúde Pública, v.42, n.5, p.859-867, 2008.

VERAS M.A.S.M. et al. The "AMA-Brazil" cooperative Project: a nation-wide assessment of the clinical and epidemiological profile of AIDS-related deaths in Brazil in the anti-retroviral treatment era. Cadernos de Saúde Pública, v.27, suppl.1, p.104-113, 2011.

Recebido para publicação em agosto de 2012

Versão final em dezembro de 2013

Conflito de interesse: não houve

suporte financeiro: inexistente 